\title{
The Ramsey number of loose paths in 3-uniform hypergraphs
}

\author{
Leila Maherani \\ Department of Mathematical Sciences \\ Isfahan University of Technology \\ Isfahan, 84156-83111, Iran \\ 1.maherani@math.iut.ac.ir
}

\author{
Golam Reza Omidi ${ }^{1}$ \\ Department of Mathematical Sciences \\ Isfahan University of Technology \\ Isfahan, 84156-83111, Iran
}

\author{
and \\ School of Mathematics \\ Institute for Research in Fundamental Sciences \\ Tehran, 19395-5746, Iran \\ romidi@cc.iut.ac.ir
}

\section{Ghaffar Raeisi ${ }^{2}$}

Department of Mathematical Sciences

Shahrekord University

Shahrekord, P.O.Box 115, Iran

and

Maryam Shahsiah

Department of Mathematical Sciences

Isfahan University of Technology

Isfahan, 84156-83111, Iran

m.shahsiah@math.iut.ac.ir

School of Mathematics

Institute for Research in Fundamental Sciences

Tehran, 19395-5746, Iran

g.raeisi@sci.sku.ac.ir

Submitted: Sep 18, 2012; Accepted: Jan 12, 2013; Published: Jan 21, 2013

Mathematics Subject Classifications: 05C15, 05C55, 05C65.

\begin{abstract}
Recently, asymptotic values of 2-color Ramsey numbers for loose cycles and also loose paths were determined. Here we determine the 2-color Ramsey number of 3-uniform loose paths when one of the paths is significantly larger than the other: for every $n \geqslant\left\lfloor\frac{5 m}{4}\right\rfloor$, we show that

$$
R\left(\mathcal{P}_{n}^{3}, \mathcal{P}_{m}^{3}\right)=2 n+\left\lfloor\frac{m+1}{2}\right\rfloor .
$$

Keywords: Ramsey Number, Loose Path, Loose Cycle.

\footnotetext{
${ }^{1}$ This research is partially carried out in the IPM-Isfahan Branch and in part supported by a grant from IPM (No.91050416).

${ }^{2}$ This research was in part supported by a grant from IPM (No.91050018).
} 


\section{Introduction}

A hypergraph $\mathcal{H}$ is a pair $\mathcal{H}=(V, E)$, where $V$ is a finite nonempty set (the set of vertices) and $E$ is a collection of distinct nonempty subsets of $V$ (the set of edges). A $k$ uniform hypergraph is a hypergraph such that all its edges have size $k$. For two $k$-uniform hypergraphs $\mathcal{H}$ and $\mathcal{G}$, the Ramsey number $R(\mathcal{H}, \mathcal{G})$ is the smallest number $N$ such that, in any red-blue coloring of the edges of the complete $k$-uniform hypergraph $K_{N}^{k}$ on $N$ vertices there is either a red copy of $\mathcal{H}$ or a blue copy of $\mathcal{G}$. There are several natural definitions for a cycle and a path in a uniform hypergraph. Here we consider the one called loose. A $k$-uniform loose cycle $\mathcal{C}_{n}^{k}$ (shortly, a cycle of length $n$ ), is a hypergraph with vertex set $\left\{v_{1}, v_{2}, \ldots, v_{n(k-1)}\right\}$ and with the set of $n$ edges $e_{i}=\left\{v_{1}, v_{2}, \ldots, v_{k}\right\}+i(k-1)$, $i=0,1, \ldots, n-1$, where we use $\bmod n(k-1)$ arithmetic and adding a number $t$ to a set $H=\left\{v_{1}, v_{2}, \ldots, v_{k}\right\}$ means a shift, i.e. the set obtained by adding $t$ to subscripts of each element of $H$. Similarly, a $k$-uniform loose path $\mathcal{P}_{n}^{k}$ (simply, a path of length $n)$, is a hypergraph with vertex set $\left\{v_{1}, v_{2}, \ldots, v_{n(k-1)+1}\right\}$ and with the set of $n$ edges $e_{i}=\left\{v_{1}, v_{2}, \ldots, v_{k}\right\}+i(k-1), i=0,1, \ldots, n-1$ and we denote this path by $e_{0} e_{1} \cdots e_{n-1}$. For $k=2$ we get the usual definitions of a cycle and a path. In this case, a classical result in graph theory (see [1]) states that $R\left(P_{n}, P_{m}\right)=n+\left\lfloor\frac{m+1}{2}\right\rfloor$, where $n \geqslant m \geqslant 1$. Moreover, the exact values of $R\left(P_{n}, C_{m}\right)$ and $R\left(C_{n}, C_{m}\right)$ for positive integers $n$ and $m$ are determined [5]. For $k=3$ it was proved in [4] that $R\left(\mathcal{C}_{n}^{3}, \mathcal{C}_{n}^{3}\right)$, and consequently $R\left(\mathcal{P}_{n}^{3}, \mathcal{P}_{n}^{3}\right)$ and $R\left(\mathcal{P}_{n}^{3}, \mathcal{C}_{n}^{3}\right)$, are asymptotically equal to $\frac{5 n}{2}$. Subsequently, Gyárfás et. al. in [3] extended this result to the $k$-uniform loose cycles and proved that $R\left(\mathcal{C}_{n}^{k}, \mathcal{C}_{n}^{k}\right)$, and consequently $R\left(\mathcal{P}_{n}^{k}, \mathcal{P}_{n}^{k}\right)$ and $R\left(\mathcal{P}_{n}^{k}, \mathcal{C}_{n}^{k}\right)$, are asymptotically equal to $\frac{1}{2}(2 k-1) n$. For small cases, Gyárfás et. al. (see [2]) proved that $R\left(\mathcal{P}_{3}^{k}, \mathcal{P}_{3}^{k}\right)=R\left(\mathcal{P}_{3}^{k}, \mathcal{C}_{3}^{k}\right)=R\left(\mathcal{C}_{3}^{k}, \mathcal{C}_{3}^{k}\right)+1=3 k-1$ and $R\left(\mathcal{P}_{4}^{k}, \mathcal{P}_{4}^{k}\right)=R\left(\mathcal{P}_{4}^{k}, \mathcal{C}_{4}^{k}\right)=R\left(\mathcal{C}_{4}^{k}, \mathcal{C}_{4}^{k}\right)+1=4 k-2$. To see a survey on Ramsey numbers involving cycles see $[6]$.

It is easy to see that $N=(k-1) n+\left\lfloor\frac{m+1}{2}\right\rfloor$ is a lower bound for the Ramsey number $R\left(\mathcal{P}_{n}^{k}, \mathcal{P}_{m}^{k}\right)$. To show this, partition the vertex set of $\mathcal{K}_{N-1}^{k}$ into parts $A$ and $B$, where $|A|=(k-1) n$ and $|B|=\left\lfloor\frac{m+1}{2}\right\rfloor-1$, color all edges that contain a vertex of $B$ blue, and the rest red. Now, this coloring can not contain a red copy of $\mathcal{P}_{n}^{k}$, since such a copy has $(k-1) n+1$ vertices. Clearly the longest blue path has length at most $m-1$, which proves our claim. Using the same argument we can see that $N$ and $N-1$ are the lower bounds for $R\left(\mathcal{P}_{n}^{k}, \mathcal{C}_{m}^{k}\right)$ and $R\left(\mathcal{C}_{n}^{k}, \mathcal{C}_{m}^{k}\right)$, respectively. In [2], motivated by the above facts and some other results, the authors conjectured that these lower bounds give the exact values of the mentioned Ramsey numbers for $k=3$. In this paper, we consider this problem and we prove that $R\left(\mathcal{P}_{n}^{3}, \mathcal{P}_{m}^{3}\right)=2 n+\left\lfloor\frac{m+1}{2}\right\rfloor$ for every $n \geqslant\left\lfloor\frac{5 m}{4}\right\rfloor$. Throughout the paper, for a 2-edge coloring of a uniform hypergraph $\mathcal{H}$, say red and blue, we denote by $\mathcal{F}_{\text {red }}$ and $\mathcal{F}_{\text {blue }}$ the induced hypergraph on edges of colors red and blue, respectively.

\section{Preliminaries}

In this section, we present some lemmas which are essential in the proof of the main results. 
Lemma 1. Let $n \geqslant m \geqslant 3$ and $\mathcal{K}_{(k-1) n+\left\lfloor\frac{m+1}{2}\right\rfloor}^{k}$ be 2-edge colored red and blue. If $\mathcal{C}_{n}^{k} \subseteq \mathcal{F}_{\text {red }}$, then either $\mathcal{P}_{n}^{k} \subseteq \mathcal{F}_{\text {red }}$ or $\mathcal{P}_{m}^{k} \subseteq \mathcal{F}_{\text {blue }}$.

Proof. Let $e_{i}=\left\{v_{1}, v_{2}, \ldots, v_{k}\right\}+i(k-1)(\bmod n(k-1)), i=0,1, \ldots, n-1$, be the edges of $\mathcal{C}_{n}^{k} \subseteq \mathcal{F}_{\text {red }}$ and $W=\left\{x_{1}, x_{2}, \ldots, x_{\left\lfloor\frac{m+1}{2}\right\rfloor}\right\}$ be the set of the remaining vertices. Set $e_{0}^{\prime}=\left(e_{0} \backslash\left\{v_{1}\right\}\right) \cup\left\{x_{1}\right\}$ and for $1 \leqslant i \leqslant m-1$ let

$$
e_{i}^{\prime}= \begin{cases}\left(e_{i} \backslash\left\{v_{i(k-1)+1}\right\}\right) \cup\left\{x_{\frac{i+1}{2}}\right\} & \text { if } i \text { is odd } \\ \left(e_{i} \backslash\left\{v_{(i+1)(k-1)+1}\right\}\right) \cup\left\{x_{\frac{i+2}{2}}\right\} & \text { if } i \text { is even. }\end{cases}
$$

If one of $e_{i}^{\prime}$ is red, we have a monochromatic $\mathcal{P}_{n}^{k} \subseteq \mathcal{F}_{\text {red }}$, otherwise $e_{0}^{\prime} e_{1}^{\prime} \ldots e_{m-1}^{\prime}$ form a blue $\mathcal{P}_{m}^{k}$, which completes the proof.

Let $\mathcal{P}$ be a loose path and $x, y$ be vertices which are not in $\mathcal{P}$. By a $\varpi_{\left\{v_{i}, v_{j}, v_{k}\right\}^{-}}$ configuration, we mean a copy of $\mathcal{P}_{2}^{3}$ with edges $\left\{x, v_{i}, v_{j}\right\}$ and $\left\{v_{j}, v_{k}, y\right\}$ so that $v_{l}$ 's, $l \in\{i, j, k\}$, belong to two consecutive edges of $\mathcal{P}$. The vertices $x$ and $y$ are called the end vertices of this configuration. Using this notation, we have the following lemmas.

Lemma 2. Let $n \geqslant 10, \mathcal{K}_{n}^{3}$ be 2-edge colored red and blue and $\mathcal{P}$, say in $\mathcal{F}_{\text {red }}$, be a maximum path. Let $A$ be the set of five consecutive vertices of $\mathcal{P}$. If $W=\left\{x_{1}, x_{2}, x_{3}\right\}$ is disjoint from $\mathcal{P}$, then we have a $\varpi_{S}$-configuration in $\mathcal{F}_{\text {blue }}$ with two end vertices in $W$ and $S \subseteq A$.

Proof. First let $A=e \cup e^{\prime}$ for two edges $e=\left\{v_{1}, v_{2}, v_{3}\right\}$ and $e^{\prime}=\left\{v_{3}, v_{4}, v_{5}\right\}$. Since $\mathcal{P} \subseteq \mathcal{F}_{\text {red }}$ is maximal, at least one of the edges $e_{1}=\left\{x_{1}, v_{1}, v_{2}\right\}$ and $e_{2}=\left\{v_{2}, v_{3}, x_{2}\right\}$ must be blue. If both are blue, then $e_{1} e_{2}$ is such a configuration. So first let $e_{1}$ be blue and $e_{2}$ be red. Maximality of $\mathcal{P}$ implies that at least one of the edges $e_{3}=\left\{x_{2}, v_{1}, v_{4}\right\}$ or $e_{4}=\left\{x_{3}, v_{2}, v_{5}\right\}$ is blue (otherwise, replacing $e e^{\prime}$ by $e_{3} e_{2} e_{4}$ in $\mathcal{P}$ yields a red path greater than $\mathcal{P}$, a contradiction), and clearly in each case we have a $\varpi_{S^{-}}$configuration. Now, let $e_{1}$ be red and $e_{2}$ be blue. Clearly $e_{5}=\left\{v_{2}, v_{4}, x_{3}\right\}$ is blue and $e_{2} e_{5}$ form a $\varpi_{S^{-}}$-configuration. Now let $A=\left\{v_{1}, v_{2}, \ldots, v_{5}\right\}$ where $e_{1}=\left\{x, v_{1}, v_{2}\right\}, e_{2}=\left\{v_{2}, v_{3}, v_{4}\right\}$ and $e_{3}=\left\{v_{4}, v_{5}, y\right\}$ are three consecutive edges of $\mathcal{P}$. If $\left\{x_{i}, v_{2}, v_{3}\right\}$ is a red edge for some $i \in\{1,2,3\}$, then $\left\{v_{3}, v_{4}, x_{j}\right\}$ and $\left\{v_{3}, v_{5}, x_{j}\right\}$ are blue for $j \neq i$ and so we are done. By the same argument the theorem is true if $\left\{x_{i}, v_{3}, v_{4}\right\}$ is red. Now we may assume $\left\{v_{2}, v_{3}, x_{i}\right\}$ and $\left\{v_{3}, v_{4}, x_{i}\right\}$ are blue for each $i \in\{1,2,3\}$ and so there is nothing to prove.

Lemma 3. Assume that $n \geqslant\left\lfloor\frac{5 m}{4}\right\rfloor$ and $\mathcal{K}_{2 n+\left\lfloor\frac{m+1}{2}\right\rfloor}^{3}$ is 2-edge colored red and blue. If $\mathcal{P} \subseteq \mathcal{F}_{\text {blue }}$ is a maximum path and $W,|W| \geqslant 5$, is a set of the vertices which are not covered by $\mathcal{P}$, then for every 4 consecutive edges $e_{1}, e_{2}, e_{3}, e_{4}$ of $\mathcal{P}$ either there is a $\mathcal{P}_{5}^{3} \subseteq \mathcal{F}_{\text {red }}$, say $Q$, between $\left\{e_{1}, e_{2}, e_{3}, e_{4}\right\}$ and $W$ with end vertices in $W$ and with no the last vertex of $e_{4}$ as a vertex such that $|W \cap V(Q)| \leqslant 5$ or there is a $\mathcal{P}_{4}^{3} \subseteq \mathcal{F}_{\text {red }}$, say $Q$, between $\left\{e_{1}, e_{2}, e_{3}\right\}$ and $W$ with end vertices in $W$ and with no the last vertex of $e_{3}$ as a vertex such that $|W \cap V(Q)| \leqslant 4$. In each of the above cases, each vertex of $W$ except one vertex can be considered as the end vertex of $Q$. 
Proof. Suppose that $e_{1}, e_{2}, e_{3}, e_{4}$ be four consecutive edges in $\mathcal{P}$. Let $e_{i}=\left\{v_{2 i-1}, v_{2 i}, v_{2 i+1}\right\}$, $1 \leqslant i \leqslant 4$, and $W=\left\{x_{1}, \ldots, x_{t}\right\}$ and $T=\{1,2, \cdots, t\}$.

Case 1. For every $1 \leqslant i, j \leqslant t,\left\{v_{1}, v_{2}, x_{i}\right\}$ and $\left\{v_{2}, v_{3}, x_{j}\right\}$ are red.

Subcase 1 . For every $1 \leqslant k, l \leqslant t$, the edges $\left\{v_{3}, v_{4}, x_{k}\right\}$ and $\left\{v_{4}, v_{5}, x_{l}\right\}$ are red.

For each $\left\{i_{1}, i_{2}, i_{3}, i_{4}\right\} \in P_{4}(T)$, edges, $\left\{x_{i_{1}}, v_{1}, v_{2}\right\},\left\{v_{2}, x_{i_{2}}, v_{3}\right\},\left\{v_{3}, x_{i_{3}}, v_{4}\right\},\left\{v_{4}, v_{5}, x_{i_{4}}\right\}$ make a red $\mathcal{P}_{4}^{3}$ with end vertices $x_{i_{1}}$ and $x_{i_{4}}$.

Subcase 2. There exists $1 \leqslant k \leqslant t$, such that the edge $\left\{v_{3}, v_{4}, x_{k}\right\}$ is blue.

So for each $\left\{i_{1}, i_{2}, i_{3},\right\} \in P_{3}(T)$ with $k \neq i_{2}, i_{3},\left\{x_{i_{1}}, v_{1}, v_{2}\right\},\left\{v_{2}, v_{3}, x_{i_{2}}\right\},\left\{x_{i_{2}}, v_{5}, v_{4}\right\}$, $\left\{v_{4}, v_{6}, x_{i_{3}}\right\}$ are the edges of a red desired $\mathcal{P}_{4}^{3}$ with end vertices $x_{i_{1}}$ and $x_{i_{3}}$.

Subcase 3. There exists $1 \leqslant k \leqslant t$, such that the edge $\left\{v_{4}, v_{5}, x_{k}\right\}$ is blue.

If for every $1 \leqslant i, j \leqslant t$, the edges $\left\{v_{5}, v_{6}, x_{i}\right\}$ and $\left\{v_{6}, v_{7}, x_{j}\right\}$ are red, then for every $\left\{i_{1}, i_{2}, i_{3}, i_{4}\right\} \in P_{4}(T)$ with $i_{3} \neq k$, we can find a red copy of $\mathcal{P}_{5}^{3}$ with edges $\left\{x_{i_{1}}, v_{1}, v_{2}\right\},\left\{v_{2}, x_{i_{2}}, v_{3}\right\},\left\{v_{3}, v_{4}, x_{i_{3}}\right\},\left\{x_{i_{3}}, v_{5}, v_{6}\right\},\left\{v_{6}, v_{7}, x_{i_{4}}\right\}$ and end vertices $x_{i_{1}}$ and $x_{i_{4}}$. Otherwise there exists $1 \leqslant l \leqslant t$, such that either $\left\{v_{5}, v_{6}, x_{l}\right\}$ or $\left\{v_{6}, v_{7}, x_{l}\right\}$ is blue. For the first one, for every $\left\{i_{1}, i_{2}, i_{3}, i_{4}\right\} \in P_{4}(T)$ with $i_{3} \neq k, l$ and $i_{4} \neq l$, $\left\{x_{i_{1}}, v_{1}, v_{2}\right\},\left\{v_{2}, x_{i_{2}}, v_{3}\right\},\left\{v_{3}, v_{4}, x_{i_{3}}\right\},\left\{x_{i_{3}}, v_{7}, v_{6}\right\},\left\{v_{6}, v_{8}, x_{i_{4}}\right\}$ make a red copy of $\mathcal{P}_{5}^{3}$ with end vertices $x_{i_{1}}$ and $x_{i_{4}}$ and for the second one, for every $\left\{i_{1}, i_{2}, i_{3}\right\} \in P_{3}(T)$ with $l \neq i_{2}, i_{3}$ the edges, $\left\{x_{i_{1}}, v_{1}, v_{2},\right\},\left\{v_{2}, v_{3}, x_{i_{2}}\right\},\left\{x_{i_{2}}, v_{6}, v_{5}\right\},\left\{v_{5}, x_{i_{3}}, x_{l}\right\}$ make a red $\mathcal{P}_{4}^{3}$ with end vertices $x_{i_{1}}$ and $y$ where $y \in\left\{x_{i_{3}}, x_{l}\right\}$.

Case 2. For some $1 \leqslant i \leqslant t,\left\{v_{1}, v_{2}, x_{i}\right\}$ is blue.

Subcase 1. For every $1 \leqslant k, l \leqslant t$, the edges $\left\{v_{5}, v_{6}, x_{k}\right\}$ and $\left\{v_{6}, v_{7}, x_{l}\right\}$ are red.

For each $\left\{i_{1}, i_{2}, i_{3}, i_{4}\right\} \in P_{4}(T)$ with $i_{j} \neq i, 1 \leqslant j \leqslant 4$, the edges, $\left\{x_{i_{1}}, x_{i}, v_{3}\right\}$, $\left\{v_{3}, x_{i_{2}}, v_{2}\right\},\left\{v_{2}, v_{4}, x_{i_{3}}\right\},\left\{x_{i_{3}}, v_{5}, v_{6}\right\},\left\{v_{6}, v_{7}, x_{i_{4}}\right\}$ make a red $\mathcal{P}_{5}^{3}$ with end vertices $y$, $y \in\left\{x_{i_{1}}, x_{i}\right\}$, and $x_{i_{4}}$.

Subcase 2. For some $1 \leqslant k \leqslant t,\left\{v_{5}, v_{6}, x_{k}\right\}$ is blue.

In this case, for each $\left\{i_{1}, i_{2}, i_{3}, i_{4}\right\} \in P_{4}(T)$ with $i_{j} \neq i, 1 \leqslant j \leqslant 4$, and $i_{3}, i_{4} \neq k$, the edges $\left\{x_{i_{1}}, x_{i}, v_{3}\right\},\left\{v_{3}, x_{i_{2}}, v_{2}\right\},\left\{v_{2}, v_{4}, x_{i_{3}}\right\},\left\{x_{i_{3}}, v_{7}, v_{6}\right\},\left\{v_{6}, v_{8}, x_{i_{4}}\right\}$ make a red $\mathcal{P}_{5}^{3}$ with end vertices $y, y \in\left\{x_{i_{1}}, x_{i}\right\}$, and $x_{i_{4}}$.

Subcase 3. For some $1 \leqslant k \leqslant t,\left\{v_{6}, v_{7}, x_{k}\right\}$ is blue.

In this case, for each $\left\{i_{1}, i_{2}, i_{3}\right\} \in P_{3}(T)$ with $i_{j} \neq i, 1 \leqslant j \leqslant 3$, and $i_{2}, i_{3} \neq k$, the edges $\left\{x_{i_{1}}, x_{i}, v_{3}\right\},\left\{v_{3}, v_{2}, x_{i_{2}}\right\},\left\{x_{i_{2}}, v_{4}, v_{6}\right\},\left\{v_{6}, v_{5}, x_{i_{3}}\right\}$ make a red $\mathcal{P}_{4}^{3}$ with end vertices $y$, $y \in\left\{x_{i_{1}}, x_{i}\right\}$, and $x_{i_{3}}$.

Case 3. For some $1 \leqslant i \leqslant t,\left\{v_{2}, v_{3}, x_{i}\right\}$ is blue.

Subcase 1 . For every $1 \leqslant k, l \leqslant t$, the edges $\left\{v_{3}, v_{4}, x_{k}\right\}$ and $\left\{v_{4}, v_{5}, x_{l}\right\}$ are red. 
For each $\left\{i_{1}, i_{2}, i_{3}\right\} \in P_{3}(T)$ with $i_{j} \neq i, 1 \leqslant j \leqslant 3,\left\{x_{i_{1}}, x_{i}, v_{1}\right\},\left\{v_{1}, v_{2}, x_{i_{2}}\right\}$, $\left\{x_{i_{2}}, v_{3}, v_{4}\right\},\left\{v_{4}, v_{5}, x_{i_{3}}\right\}$ are the edges of a red $\mathcal{P}_{4}^{3}$ with end vertices $y, y \in\left\{x_{i_{1}}, x_{i}\right\}$, and $x_{i_{3}}$.

Subcase 2. For some $1 \leqslant k \leqslant t,\left\{v_{3}, v_{4}, x_{k}\right\}$ is blue.

In this case, for each $\left\{i_{1}, i_{2}, i_{3}\right\} \in P_{3}(T)$ with $i_{j} \neq i, 1 \leqslant j \leqslant 3$, and $i_{2}, i_{3} \neq k$, the edges, $\left\{x_{i_{1}}, x_{i}, v_{1}\right\},\left\{v_{1}, v_{2}, x_{i_{2}}\right\},\left\{x_{i_{2}}, v_{5}, v_{4}\right\},\left\{v_{4}, v_{6}, x_{i_{3}}\right\}$ make a red copy of $\mathcal{P}_{4}^{3}$ with end vertices $y, y \in\left\{x_{i_{1}}, x_{i}\right\}$, and $x_{i_{3}}$.

Subcase 3. For some $1 \leqslant k \leqslant t,\left\{v_{4}, v_{5}, x_{k}\right\}$ is blue.

If for every $1 \leqslant l, h \leqslant t$, the edges $\left\{v_{5}, v_{6}, x_{l}\right\}$ and $\left\{v_{6}, v_{7}, x_{h}\right\}$ are red, then for each $\left\{i_{1}, i_{2}, i_{3}, i_{4}\right\} \in P_{4}(T)$ with $i_{j} \neq i, 1 \leqslant j \leqslant 4$, and $i_{3} \neq k$, the edges, $\left\{x_{i_{1}}, x_{i}, v_{1}\right\},\left\{v_{1}, x_{i_{2}}, v_{2}\right\},\left\{v_{2}, v_{4}, x_{i_{3}}\right\},\left\{x_{i_{3}}, v_{5}, v_{6}\right\},\left\{v_{6}, v_{7}, x_{i_{4}}\right\}$ make a red $\mathcal{P}_{5}^{3}$ with end vertices $y, y \in\left\{x_{i_{1}}, x_{i}\right\}$, and $x_{i_{4}}$. Otherwise there exists $1 \leqslant l \leqslant t$, such that either $\left\{v_{5}, v_{6}, x_{l}\right\}$ or $\left\{v_{6}, v_{7}, x_{l}\right\}$ is blue. For the first one, for each $\left\{i_{1}, i_{2}, i_{3}, i_{4}\right\} \in P_{4}(T)$ with $i_{j} \neq i, 1 \leqslant j \leqslant 4, i_{3} \neq k, l$ and $i_{4} \neq l$, the edges $\left\{x_{i_{1}}, x_{i}, v_{1}\right\},\left\{v_{1}, x_{i_{2}}, v_{2}\right\},\left\{v_{2}, v_{4}, x_{i_{3}}\right\}$, $\left\{x_{i_{3}}, v_{7}, v_{6}\right\},\left\{v_{6}, v_{8}, x_{i_{4}}\right\}$ make a red copy of $\mathcal{P}_{5}^{3}$ with end vertices $y, y \in\left\{x_{i_{1}}, x_{i}\right\}$ and $x_{i_{4}}$. For the second one, for every $\left\{i_{1}, i_{2}, i_{3}\right\} \in P_{3}(T)$ with $i_{j} \neq i, 1 \leqslant j \leqslant 3$, and $i_{2}, i_{3} \neq l$, $\left\{\left\{x_{i_{1}}, x_{i}, v_{1}\right\},\left\{v_{1}, v_{2}, x_{i_{2}}\right\},\left\{x_{i_{2}}, v_{4}, v_{6}\right\},\left\{v_{6}, v_{5}, x_{i_{3}}\right\}\right\}$ is the set of the edges of a red $\mathcal{P}_{4}^{3}$ with end vertices $y, y \in\left\{x_{i_{1}}, x_{i}\right\}$, and $x_{i_{3}}$. These observations complete the proof.

\section{Main Results}

In this section, we prove that $R\left(\mathcal{P}_{n}^{3}, \mathcal{P}_{m}^{3}\right)=2 n+\left\lfloor\frac{m+1}{2}\right\rfloor$ for every $n \geqslant\left\lfloor\frac{5 m}{4}\right\rfloor$. First we present several lemmas which will be our main tools in establishing the main theorem.

Lemma 4. Assume that $n=\left\lfloor\frac{5 m}{4}\right\rfloor$ and $\mathcal{K}_{2 n+\left\lfloor\frac{m+1}{2}\right\rfloor}^{3}$ is 2-edge colored red and blue. If $\mathcal{P}=\mathcal{P}_{m-1}^{3}$ is a maximum blue path, then $\mathcal{P}_{n-1}^{3} \subseteq \stackrel{\mathcal{F}}{\text { red }}^{2}$.

Proof. Let $t=2 n+\left\lfloor\frac{m+1}{2}\right\rfloor$ and $\mathcal{P}=e_{1} e_{2} \ldots e_{m-1}$ be a copy of $\mathcal{P}_{m-1}^{3} \subseteq \mathcal{F}_{\text {blue }}$ with edges $e_{i}=\left\{v_{1}, v_{2}, v_{3}\right\}+2(i-1), i=1, \ldots, m-1$. Set $W=V\left(\mathcal{K}_{t}^{3}\right) \backslash V(\mathcal{P})$. Using Lemma 3 there is a red path $Q_{1}$ with end vertices $x_{1}$ and $y_{1}$ in $W_{1}=W$ between $E_{1}^{\prime}$ and $W_{1}$ where $E_{1}=\left\{e_{i}: i_{1}=1 \leqslant i \leqslant 4\right\}, \bar{E}_{1}=E_{1} \backslash\left\{e_{4}\right\}$ and $E_{1}^{\prime} \in\left\{E_{1}, \bar{E}_{1}\right\}$. Set $i_{2}=\min \left\{j: j \in\left\{i_{1}+3, i_{1}+4\right\}, e_{j} \notin E_{1}^{\prime}\right\}, E_{2}=\left\{e_{i}: i_{2} \leqslant i \leqslant i_{2}+3\right\}$ and $\bar{E}_{2}=E_{2} \backslash\left\{e_{i_{2}+3}\right\}$ and $W_{2}=(W \backslash V(Q)) \cup\left\{x_{1}, y_{1}\right\}$. Again using Lemma 3 there is a red path $Q_{2}$ between $E_{2}^{\prime}$ and $W_{2}$ such that $Q_{1} \cup Q_{2}$ is a red path with end vertices $x_{2}, y_{2}$ in $W_{2}$ where $E_{2}^{\prime} \in\left\{E_{2}, \bar{E}_{2}\right\}$ and again set $i_{3}=\min \left\{j: j \in\left\{i_{2}+3, i_{2}+4\right\}, e_{j} \notin E_{2}^{\prime}\right\}, E_{3}=\left\{e_{i}: i_{3} \leqslant i \leqslant i_{3}+3\right\}$, $\bar{E}_{3}=E_{3} \backslash\left\{e_{i_{3}+3}\right\}$ and $W_{3}=\left(W \backslash V\left(Q_{1} \cup Q_{2}\right)\right) \cup\left\{x_{2}, y_{2}\right\}$. Since $|W| \geqslant m$, using Lemma 3 by continuing the above process we can partition $E(\mathcal{P}) \backslash\left\{e_{m-1}\right\}$ into classes $E_{i}^{\prime}$ th, $\left|E_{i}^{\prime}\right| \in\{3,4\}$ and at most one class of size $r \leqslant 3$ of the last edges such that for each $i$, there is a red $Q_{i}=\mathcal{P}_{5}^{3}\left(\right.$ resp. $\left.Q_{i}=\mathcal{P}_{4}^{3}\right)$ between $E_{i}^{\prime}$ and $W$ with the properties in Lemma 3 if $\left|E_{i}^{\prime}\right|=4$ (resp. $\left|E_{i}^{\prime}\right|=3$ ) and $\mathcal{P}^{\prime}=\cup Q_{i}$ is a red path with end vertices $x, y$ in $W$. Let 
$l_{1}=\left|\left\{i:\left|E_{i}^{\prime}\right|=4\right\}\right|$ and $l_{2}=\left|\left\{i:\left|E_{i}^{\prime}\right|=3\right\}\right|$. So $m-2=4 l_{1}+3 l_{2}+r, 0 \leqslant r \leqslant 3$ and $\mathcal{P}^{\prime}$ has $5 l_{1}+4 l_{2}$ edges. One can easily check that $5 l_{1}+4 l_{2} \geqslant \frac{5}{4}(m-2-r)$. Also we have

$$
\left|W \cap V\left(\mathcal{P}^{\prime}\right)\right| \leqslant 4 l_{1}+3 l_{2}+1=m-1-r .
$$

Let $T=V\left(\mathcal{K}_{t}^{3}\right) \backslash\left(V(\mathcal{P}) \cup V\left(\mathcal{P}^{\prime}\right)\right)$ and suppose that $m=4 k+p$ for some $p, 0 \leqslant p \leqslant 4$. Therefore $|T| \geqslant r+2$ if $p=0,1$ and $|T| \geqslant r+1$ if $p=2,3$. Now we consider the following cases.

Case 1. $r=0$.

Clearly $|T| \geqslant 1$ and it is easy to see that $\mathcal{P}^{\prime}$ contains at least $n-2$ edges. Let $\{u\} \subseteq T$. The maximality of $\mathcal{P}$ implies that the edge $e=\left\{v_{2 m-1}, x, u\right\}$ is red and hence $\mathcal{P}^{\prime} \cup\{e\}$ is a red copy of $\mathcal{P}_{n-1}^{3}$.

Case 2. $r=1$.

In this case, $|T| \geqslant 2$ and it is easy to see that $\mathcal{P}^{\prime}$ contains at least $n-3$ edges. Let $\{u, v\} \subseteq T$. Clearly $\mathcal{P}^{\prime} \cup\left\{\left\{v_{2 m-2}, x, u\right\},\left\{v_{2 m-1}, u, v\right\}\right\}$ is a red copy of $\mathcal{P}_{n-1}^{3}$.

Case 3. $r=2$.

It is easily seen that $|T| \geqslant 3$ and $\mathcal{P}^{\prime}$ contains at least $n-5$ edges. Let $T^{\prime}=\{u, v, w\} \subseteq$ $T$. Since $V\left(\mathcal{P}^{\prime}\right) \cap V\left(e_{m-3} \cup e_{m-2}\right)=\emptyset$ by Lemma 2 there is a red $\varpi_{S}$-configuration with $S \subset e_{m-3} \cup e_{m-2}$ and its end vertices in $T^{\prime}$, say $u$ and $v$. The maximality of $\mathcal{P}$ implies that the edges $\left\{v_{2 m-2}, x, u\right\}$ and $\left\{v_{2 m-1}, v, w\right\}$ are red and clearly we have a red $\mathcal{P}_{n-1}^{3}$.

Case 4. $r=3$.

In this case, for $p \in\{2,3\}$ we have $|T| \geqslant 4$ and $\mathcal{P}^{\prime}$ contains at least $n-5$ edges. Using an argument similar to case 3 we can complete the proof. Now let $p \in\{0,1\}$. Then $|T| \geqslant 5$ and $\mathcal{P}^{\prime}$ contains at least $n-6$ edges. Set $T^{\prime}=\{u, v, w, z, t\} \subseteq T$. By Lemma 2, there is a $\varpi_{S^{-}}$configuration $C$ with $S \subseteq V\left(e_{m-3} \cup e_{m-2}\right)$ and end vertices in $T^{\prime}$, say $u$ and $v$. Clearly $\mathcal{P}^{\prime} \cup\left\{\left\{y, w, v_{2 m-2}\right\},\left\{v_{2 m-2}, z, t\right\},\left\{v_{2 m-1}, t, u\right\}\right\} \cup C$ is a red $\mathcal{P}_{n-1}^{3}$. These observations complete the proof.

Lemma 5. Let $n \geqslant\left\lfloor\frac{5 m}{4}\right\rfloor$ and $\mathcal{K}_{2 n+\left\lfloor\frac{m+1}{2}\right\rfloor}^{3}$ be 2-edge colored red and blue. If $\mathcal{P}_{n-1}^{3} \subseteq \mathcal{F}_{\text {red }}$ be a maximum path, then $\mathcal{P}_{m}^{3} \subseteq \mathcal{F}_{\text {blue }}$.

Proof. Let $t=2 n+\left\lfloor\frac{m+1}{2}\right\rfloor$ and $\mathcal{P}=e_{1} e_{2} \ldots e_{n-1}$ be a copy of $\mathcal{P}_{n-1}^{3} \subseteq \mathcal{F}_{\text {red }}$ with end edges $e_{1}=\left\{v_{1}, v_{2}, v_{3}\right\}$ and $e_{n-1}=\left\{v_{2 n-3}, v_{2 n-2}, v_{2 n-1}\right\}$. By Lemma 1 , we may assume that the subhypergraph induced by $V(\mathcal{P})$ does not have a red copy of $\mathcal{C}_{n}^{3}$. Let $W=V\left(\mathcal{K}_{t}^{3}\right) \backslash V(\mathcal{P})$ and let $2 n-2=5 q+h$ where $0 \leqslant h<5$. Partition the set $V(\mathcal{P}) \backslash\left\{v_{1}\right\}$ into $q$ classes $A_{1}, A_{2}, \ldots, A_{q}$ of size five and one class $A_{q+1}=\left\{v_{2 n-h}, \ldots, v_{2 n-2}, v_{2 n-1}\right\}$ of size $h$ if $h>0$, so that each class contains consecutive vertices of $\mathcal{P}$. Using Lemma 2, there is a blue $\varpi_{S_{1}}$-configuration, $\bar{c}_{1}$, with the set of end vertices $E_{1} \subseteq W$ and $S_{1} \subseteq A_{1}$. Let $x_{1} \in E_{1}$ and $B_{1}$ be a 2 -subset of $W \backslash E_{1}$. Again by Lemma 2 , there is a blue $\varpi_{S_{2}}$-configuration, $\bar{c}_{2}$, with 
the set of end vertices $E_{2} \subseteq\left(B_{1} \cup\left\{x_{1}\right\}\right)$ and $S_{2} \subseteq A_{2}$. If $x_{1} \notin E_{2}$, then let $\bar{c}_{3}$ be a blue $\varpi_{S_{3}}$-configuration with the set of end vertices $E_{3} \subseteq\left\{x_{1}, y, z\right\}$ and $S_{3} \subseteq A_{3}$ where $y \in B_{1}$ and $z \in W \backslash\left(E_{1} \cup E_{2}\right)$. If $x_{1} \in E_{2}$, then let $\bar{c}_{3}$ be a blue $\varpi_{S_{3}}$-configuration with the set of end vertices $E_{3} \subseteq\left\{x_{2}, y, z\right\}$ and $S_{3} \subseteq A_{3}$ where $x_{2} \in E_{2} \backslash\left\{x_{1}\right\}$ and $\{y, z\} \subseteq W \backslash\left(E_{1} \cup E_{2}\right)$. We continue this process to find the set of $\left\{\bar{c}_{1}, \bar{c}_{2}, \ldots, \bar{c}_{q^{\prime}}\right\}$ of configurations. When this process terminate, we have the paths $\mathcal{P}_{l^{\prime \prime}}$ and $\mathcal{P}_{l^{\prime}}$ where $l^{\prime \prime} \geqslant l^{\prime} \geqslant 0$ and $l^{\prime \prime}+l^{\prime}=2 q^{\prime}$. Let $x^{\prime \prime}, y^{\prime \prime}$ (resp. $x^{\prime}, y^{\prime}$ if $l^{\prime}>0$ ) be the end vertices of $\mathcal{P}_{l^{\prime \prime}}$ (resp. $\mathcal{P}_{l^{\prime}}$ ) in $W$. Let $T=V\left(\mathcal{K}_{t}^{3}\right) \backslash\left(V(\mathcal{P}) \cup V\left(\mathcal{P}_{l^{\prime \prime}}\right) \cup V\left(\mathcal{P}_{l^{\prime}}\right)\right)$. Clearly $|T|=\left\lfloor\frac{m+1}{2}\right\rfloor+1-\left(q^{\prime}+i\right)$ where $i=1$ if $l^{\prime}=0$ and $i=2$ if $l^{\prime}>0$. Assume $m=4 k+r$ for some $r, 0 \leqslant r \leqslant 3$. We have the following cases.

Case 1. $r=0$.

Since $q \geqslant 2 k-1$, we have $2 q^{\prime} \geqslant m-2$. On the other hand, $|W|=\left\lfloor\frac{m+1}{2}\right\rfloor+1$ and so $2 q^{\prime} \leqslant m$. If $2 q^{\prime}=m$, then $l^{\prime}=0$ and so $\mathcal{P}_{l^{\prime \prime}=m}$ is a blue path. Now we may assume that $2 q^{\prime}=m-2$, and one can easily check that the vertices $\left\{v_{2 n-3}, v_{2 n-2}, v_{2 n-1}\right\}$ are not used in $\mathcal{P}_{l^{\prime \prime}} \cup \mathcal{P}_{l^{\prime}}$. First let $l^{\prime}=0$. Then $|T|=1$ and we may assume $T=\{u\}$. Now using the maximality of $\mathcal{P}$ and the fact that $\mathcal{C}_{n}^{3} \nsubseteq \mathcal{F}_{\text {red }}, \mathcal{P}_{l^{\prime \prime}} \cup\left\{\left\{v_{2 n-2}, y^{\prime \prime}, u\right\},\left\{v_{2 n-1}, u, v_{1}\right\}\right\}$ is a blue $\mathcal{P}_{m}^{3}$. For $l^{\prime}>0, \mathcal{P}_{l^{\prime \prime}} \cup\left\{\left\{v_{2 n-2}, y^{\prime \prime}, x^{\prime}\right\}\right\} \cup \mathcal{P}_{l^{\prime}} \cup\left\{\left\{v_{2 n-1}, y^{\prime}, v_{1}\right\}\right\}$ is a blue $\mathcal{P}_{m}^{3}$.

Case 2. $r=1$.

Since $|W|=\left\lfloor\frac{m+1}{2}\right\rfloor+1,2 q^{\prime} \leqslant m+1$ and if the equality holds, then $l^{\prime}=0$. On the other hand, $q \geqslant 2 k$ and so $2 q^{\prime} \geqslant m-1$. Hence $2 q^{\prime} \in\{m+1, m-1\}$. If $2 q^{\prime}=m+1$, then $l^{\prime}=0$ and there is a blue $\mathcal{P}_{m+1}^{3}$. Now let $2 q^{\prime}=m-1$. If $l^{\prime}=0$, then $|T|=1$, so $T=\{u\}$ and hence $\mathcal{P}_{l^{\prime \prime}} \cup\left\{\left\{v_{1}, u, y^{\prime \prime}\right\}\right\}$ is a blue $\mathcal{P}_{m}^{3}$. If $l^{\prime}>0$, then $\mathcal{P}_{l^{\prime \prime}} \cup\left\{\left\{v_{1}, y^{\prime \prime}, x^{\prime}\right\}\right\} \cup \mathcal{P}_{l^{\prime}}$ is a blue $\mathcal{P}_{m}^{3}$.

Case 3. $r=2$.

Using an argument similar to the case 1 , we have $2 q^{\prime} \in\{m, m-2\}$ and if $2 q^{\prime}=m$, then $l^{\prime}=0$ and we have a blue $\mathcal{P}_{l^{\prime \prime}=m}$. Again by an argument similar to the case 1 we have a blue $\mathcal{P}_{m}^{3}$.

Case 4. $r=3$.

In this case, partition $V(\mathcal{P}) \backslash\left\{v_{1}, v_{2}\right\}$ into $\left\lfloor\frac{2 n-3}{5}\right\rfloor$ classes of size five and possibly one class of size at most four. Then we repeat the mentioned process in the first of the proof to find blue paths $\mathcal{P}_{l^{\prime \prime}}$ and $\mathcal{P}_{l^{\prime}}$ with $l^{\prime \prime} \geqslant l^{\prime} \geqslant 0$ and $l^{\prime \prime}+l^{\prime}=2 q^{\prime}$. Again using a similar argument in case 1 , we have $2 q^{\prime} \in\{m+1, m-1, m-3\}$. If $2 q^{\prime}=m+1$, then we have $l^{\prime}=0$ and so there is a blue $\mathcal{P}_{m+1}^{3}$. For $2 q^{\prime}=m-1$, the assertion holds by an argument similar to the case 2. Now let $2 q^{\prime}=m-3$. If $l^{\prime}=0$, then $|T|=2$, so $T=\{u, v\}$ and hence $\mathcal{P}_{l^{\prime \prime}} \cup\left\{\left\{v_{2 n-2}, v_{2}, y^{\prime \prime}\right\},\left\{v_{2 n-2}, v, u\right\},\left\{u, v_{1}, v_{2 n-1}\right\}\right\}$ is a blue $\mathcal{P}_{m}^{3}$ (note that $\left.\left\{v_{2 n-3}, v_{2 n-2}, v_{2 n-1}\right\} \cap V\left(\mathcal{P}_{l^{\prime \prime}}\right)=\emptyset\right)$. If $l^{\prime}>0$, then $|T|=1$, so $T=\{u\}$ and hence $\mathcal{P}_{l^{\prime \prime}} \cup\left\{\left\{v_{2 n-2}, v_{2}, y^{\prime \prime}\right\},\left\{v_{2 n-2}, x^{\prime}, u\right\}\right\} \cup \mathcal{P}_{l^{\prime}} \cup\left\{\left\{y^{\prime}, v_{1}, v_{2 n-1}\right\}\right\}$ is a blue $\mathcal{P}_{m}^{3}$ and the proof is completed. 
Theorem 6. For every $n \geqslant\left\lfloor\frac{5 m}{4}\right\rfloor$,

$$
R\left(\mathcal{P}_{n}^{3}, \mathcal{P}_{m}^{3}\right)=2 n+\left\lfloor\frac{m+1}{2}\right\rfloor
$$

Proof. We prove the theorem by induction on $m+n$. The proof of the case $m=n=1$ is trivial. Suppose that for $m^{\prime}+n^{\prime}<m+n$ with $n^{\prime} \geqslant\left\lfloor\frac{5 m^{\prime}}{4}\right\rfloor, R\left(\mathcal{P}_{n^{\prime}}^{3}, \mathcal{P}_{m^{\prime}}^{3}\right)=2 n^{\prime}+\left\lfloor\frac{m^{\prime}+1}{2}\right\rfloor$. Now, let $n \geqslant\left\lfloor\frac{5 m}{4}\right\rfloor$ and let $\mathcal{K}_{2 n+\left\lfloor\frac{m+1}{2}\right\rfloor}^{3}$ be 2-edge colored red and blue. We may assume there is no red copy of $\mathcal{P}_{n}^{3}$ and no blue copy of $\mathcal{P}_{m}^{3}$. Consider the following cases.

Case 1. $n=\left\lfloor\frac{5 m}{4}\right\rfloor$.

Since $R\left(\mathcal{P}_{n-1}^{3}, \mathcal{P}_{m-1}^{3}\right)=2(n-1)+\left\lfloor\frac{m}{2}\right\rfloor<2 n+\left\lfloor\frac{m+1}{2}\right\rfloor$ by induction hypothesis, then either there is a $\mathcal{P}_{n-1}^{3} \subseteq \mathcal{F}_{\text {red }}$ or a $\mathcal{P}_{m-1}^{3} \subseteq \mathcal{F}_{\text {blue }}$. If we have a red copy of $\mathcal{P}_{n-1}^{3}$, then by Lemma 5 we have a $\mathcal{P}_{m}^{3} \subseteq \mathcal{F}_{\text {blue }}$. Now assume that there is a blue copy of $\mathcal{P}_{m-1}^{3}$. Lemma 4 implies that $\mathcal{P}_{n-1}^{3} \subseteq \mathcal{F}_{\text {red }}$ and using Lemma 5 we have $\mathcal{P}_{m}^{3} \subseteq \mathcal{F}_{\text {blue }}$, a contradiction.

Case 2. $n>\left\lfloor\frac{5 m}{4}\right\rfloor$.

In this case, $n-1 \geqslant\left\lfloor\frac{5 m}{4}\right\rfloor$ and since $R\left(\mathcal{P}_{n-1}^{3}, \mathcal{P}_{m}^{3}\right)=2(n-1)+\left\lfloor\frac{m+1}{2}\right\rfloor<2 n+\left\lfloor\frac{m+1}{2}\right\rfloor$, by induction hypothesis we have a $\mathcal{P}_{n-1}^{3} \subseteq \mathcal{F}_{\text {red }}$. Using Lemma 5 we have a $\mathcal{P}_{m}^{3} \subseteq \mathcal{F}_{\text {blue }}$ and it completes the proof.

\section{Acknowledgments}

The authors appreciate the discussions on the subject of this paper with A. Gyárfás. Also the third author would like to thanks Shahrekord University for its support.

\section{References}

[1] L. Gerencsér, A. Gyárfás, On Ramsey-type problems, Ann. Univ. Sci. Budapest, Eötvös Sect. Math. 10 (1967), 167-170.

[2] A. Gyárfás, G. Raeisi, The Ramsey number of loose triangles and quadrangles in hypergraphs, Electron. J. Combin. 19 (2012), no. 2, \#R30.

[3] A. Gyárfás, G. Sárközy, E. Szemerédi, The Ramsey number of diamond-matchings and loose cycles in hypergraphs, Electron. J. Combin. 15 (2008), no. 1, \#R126.

[4] P. Haxell, T. Łuczak, Y. Peng, V. Rödl, A. Ruciński, M. Simonovits, J. Skokan, The Ramsey number for hypergraph cycles I, J. Combin. Theory, Ser. A, 113 (2006), 67-83.

[5] S. P. Radziszowski, Small Ramsey numbers, Electron. J. Combin. 1 (1994), Dynamic Surveys, DS1.13 (August 22, 2011).

[6] S. P. Radziszowski, Ramsey numbers involving cycles, in Ramsey Theory, Yesterday, Today and Tomorrow, A. Soifer ed., Progress in Mathematics 285. 